\title{
A TUG on glucose transport
}

\author{
Alan R Saltiel \\ Glucose transport into the cell is a delicate process that is highly responsive to insulin. A newly identified protein \\ that may tether to the glucose transporter helps keep glucose traffic running smoothly in human cells.
}

One of the first defects in the development of type 2 diabetes is the failure of insulin to stimulate glucose uptake into fat and muscle cells ${ }^{1,2}$. The facilitative transporter GLUT4 mediates this process. In the basal state, GLUT4 slowly recycles among vesicular compartments, ensnared inside the cell while waiting for a signal to travel to the plasma membrane. Upon exposure to insulin, a pool of GLUT4-containing vesicles moves to the plasma membrane by targeted exocytosis, followed by docking and fusion. These events result in the extracellular exposure of GLUT4 and a marked increase in glucose uptake ${ }^{3}$.

Although there is substantial evidence that sequestered GLUT4 dwells in specialized insulin-sensitive vesicles, it is unclear where these vesicles are located, how they are retained, and how they move from place to place. Bogan et al. ${ }^{4}$ have identified a new molecular tether, TUG, that might help keep GLUT4 trapped inside cells and provide a target for insulin to trigger vesicle release and GLUT4 translocation to the cell surface.

Kinetic analyses of GLUT4 translocation to the plasma membrane and glucose uptake suggest a progression between states ${ }^{3}$. For example, the appearance of GLUT4 at the plasma membrane precedes the accessibility of its extracellular domain increases in glucose uptake. Different mechanisms appear to exist for the trafficking, targeting, docking and fusion of these vesicles ${ }^{3}$. The localization of GLUT4 into a distinct compartment could enable insulin to rapidly stimulate GLUT4 translocation to the plasma membrane, without major effects on the translocation of other recycling proteins.

For these reasons, the mechanisms of intracellular tethering of the GLUT4 vesicle in resting cells have been explored for years. Bogan et al. attacked this problem by searching for proteins that might enhance or diminish translocation of GLUT4 after only a short exposure to insulin. They identified a

Alan R. Saltiel is at the Life Sciences Institute, University of Michigan, Ann Arbor, Michigan 48109, USA.

e-mail: saltiel@med.umich.edu novel cDNA encoding TUG (tethering protein containing a UBX domain for GLUT4). The TUG cDNA was first discovered as a fragment that blocked the translocation of GLUT4 when overexpressed in cells.

Bogan et al. found that this C-terminal fragment behaved as a dominant-negative mutant, serving as a decoy for release of the tether. In contrast, overexpression of the full-length protein enhanced glucose uptake and GLUT4 translocation. The shorter form seems to result from alternate splicing of the gene encoding TUG, raising the possibility that the dynamic equilibrium of these isoforms controls the size of the insulin-sensitive pool of transporters. The molecular basis for TUG's action is unclear, although the protein has a UBX domain that presumably interacts with other protein(s) to ensure the intracellular localization of the vesicle.

How does TUG work, and what are its binding partners? Sequences in GLUT4 itself are implicated in the process of intracellular
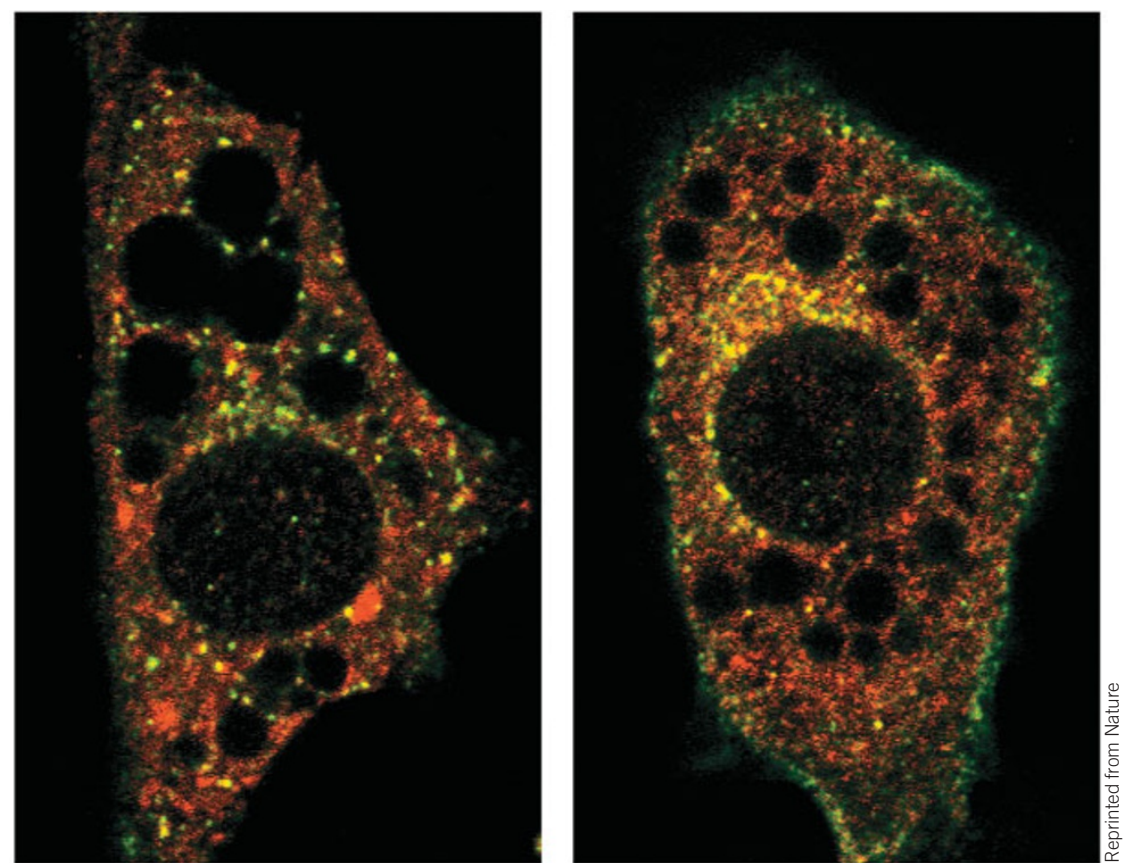

Figure 1 TUG as tether. (a) Confocal image showing punctate and perinuclear distribution of membrane-associated TUG (red). Cytosolic TUG also forms a homogenous pattern. Most TUG punctae colocalize with GLUT4. (b) After insulin addition, GLUT4 is visible at the plasma membrane, and there are fewer intracellular punctae where GLUT4 and TUG overlap. Bogan et al. suggest that insulin mobilizes a pool of GLUT4 to the membrane by releasing TUG's grip on GLUT4 (ref. 4). 